\title{
Preoperative computed tomography staging of nonmetastatic colon cancer predicts outcome: implications for clinical trials
}

\author{
NJ Smith', N Bees², Y Barbachano ${ }^{3}$, AR Norman ${ }^{3}$, RI Swift' and G Brown ${ }^{*, 4}$ \\ 'Department of Colorectal Surgery, Mayday University Hospital, Croydon, CR7 7RE, UK; ${ }^{2}$ Department of Radiology, Mayday University Hospital, Croydon, \\ CR7 7RE, UK; ${ }^{3}$ Department of Medical Statistics and Computing, Royal Marsden Hospital, Sutton, Surrey, SM2 5PT, UK; ${ }^{4}$ Department of Radiology, \\ Royal Marsden Hospital, Sutton, Surrey, SM2 5PT, UK
}

\begin{abstract}
Colon cancer patients routinely undergo preoperative computed tomography (CT) scanning, but local staging is thought to be inaccurate. We aimed to determine if clinical outcome could be predicted from radiological features of the primary tumour. Consecutive patients at one hospital undergoing primary resection for colon cancer during 2000-2004 were included. Patients with visible metastases were excluded. Preoperative CT scans were reviewed independently by two radiologists blinded to histological stage and outcome. Images of the primary tumour were evaluated according to conventional TNM criteria and patients were stratified into 'good' or 'poor' prognosis groups. Comparison was made between prognostic group and actual clinical outcome. Hundred and twenty-six preoperative CT scans were reviewed. T-stage and nodal status was correctly predicted in only 60 and $62 \%$, respectively. However, inter-observer agreement for prognostic group was 79\% $(\kappa=0.59)$ and 3-year relapse-free survival was 7 I and $43 \%$ for the CT-predicted 'good' and 'poor' groups, respectively $(P<0.0066)$. This compared favourably with 75 vs $43 \%$ for histology-predicted prognostic groups. Computed tomography is a robust method for stratifying patients preoperatively, with similar accuracy to histopathology for predicting outcome. Recognition of poor prognosis tumours preoperatively may permit investigation into the future use of neo-adjuvant therapy in colon cancer.
\end{abstract}

British Journal of Cancer (2007) 96, 1030-1036. doi:I0.1038/sj.bjc.6603646 www.bjcancer.com

Published online 13 March 2007

(c) 2007 Cancer Research UK

Keywords: colonic neoplasm; neoplasm staging; tomography; X-ray computed; prognosis; survival analysis

Colorectal adenocarcinoma is the third commonest cancer in the United Kingdom, with 35000 new cases discovered every year and 16000 deaths resulting annually (Scottish Cancer Registry, 2005; Northern Ireland Cancer Registry, 2005; Office for National Statistics, 2005; Welsh Cancer Intelligence and Surveillance Unit, 2006). Approximately $70 \%$ of cases involve the colon, the remaining 30\% involving the anus and rectum. Knowledge of the extent of the primary tumour at initial diagnosis is critical for proper management of disease as the prognosis for patients diagnosed with colon cancer is directly related to stage at presentation. In the UK, most patients with colon cancer undergo preoperative staging with abdomino-pelvic computed tomography (CT) scanning, but this is carried out in order to diagnose or exclude the presence of synchronous metastases, rather than to evaluate the characteristics of the primary tumour itself.

Primary surgery is accepted to be the only potentially curative treatment for colonic cancer. Unlike rectal cancer, where preoperative neoadjuvant therapy has been used with considerable success, there is currently no role for preoperative therapy in colon

*Correspondence: Dr G Brown; E-mail: gina.brown@rmh.nhs.uk The contents of this paper were presented to the Association of Coloproctology of Great Britain and Ireland Annual Meeting, Gateshead, 3-6th July 2006.

Received 21 September 2006; revised 24 January 2007; accepted 29 January 2007; published online 13 March 2007 cancer. Adjuvant radio- or chemotherapy may be offered postsurgery to patients with advanced-stage tumours, as defined by histological staging criteria.

Previous studies examining the usefulness of preoperative CT for staging of colon cancer have judged its 'accuracy' by comparisons with histology (Freeny et al, 1986; Balthazar et al, 1988; Acunas et al, 1990; Hundt et al, 1999). Although tumour staging has its origins in the histological description of colorectal cancers (Dukes, 1932), its rationale is in the stratification of patients into prognostic categories. It may be argued, therefore, that the value of preoperative staging should not be judged solely according to its ability to predict histology, but also according to its ability to predict outcome. We are not aware of any previous studies that have examined the correlation between CT-based staging and outcome. Therefore, we have designed a study to examine this.

\section{Aims}

1. To examine whether the radiological features of the primary colonic tumour seen on the preoperative CT scan could be used to predict clinical outcome.

2. To compare preoperative CT-based prognostication with postoperative histology (the current 'gold' standard). 
Table I Definitions of TNM components in the 6th edition of the AJCC and UICC system for staging cancer of the colon and rectum, 2002 (Sobin and Wittekind, 2002)

\begin{tabular}{|c|c|}
\hline Category & Description \\
\hline TX & The primary tumour cannot be assessed \\
\hline TO & No evidence of primary tumour \\
\hline Tis & Carcinoma in situ (intraepithelial or intramucosal carcinoma) \\
\hline $\mathrm{TI}$ & Tumour invades into the submucosa \\
\hline T2 & Tumour invades into the muscularis propria \\
\hline T3 & Tumour invades through the muscularis propria into the subserosa, or into nonperitonealised pericolic or perirectal tissues \\
\hline \multicolumn{2}{|c|}{ Optional subdivision of T3 } \\
\hline T3a & Minimal invasion: $<1 \mathrm{~mm}$ beyond the border of the muscularis propria \\
\hline $\mathrm{T} 3 \mathrm{~b}$ & Slight invasion: $1-5 \mathrm{~mm}$ beyond the border of the muscularis propria \\
\hline $\mathrm{T} 3 \mathrm{c}$ & Moderate invasion: $>5-15 \mathrm{~mm}$ beyond the border of the muscularis propria \\
\hline T3d & Extensive invasion: $>15 \mathrm{~mm}$ beyond the border of the muscularis propria \\
\hline T4 & Tumour directly invades into other organs or structures (T4a) or perforates the visceral peritoneum (T4b) \\
\hline NX & Regional lymph nodes cannot be assessed \\
\hline No & No regional lymph node metastases \\
\hline NI & Metastatic tumour in I-3 pericolic or perirectal lymph nodes \\
\hline N2 & Metastatic tumour in four or more pericolic or perirectal lymph nodes \\
\hline$M X$ & The presence of distant metastasis cannot be assessed \\
\hline MO & No distant metastasis \\
\hline MI & Distant metastasis present \\
\hline
\end{tabular}

\section{MATERIALS AND METHODS}

The medical records of all 312 consecutive patients undergoing resection of a colonic carcinoma (defined as any tumour above the peritoneal reflection) at Mayday Hospital between January 2000 and December 2004 were retrospectively reviewed. Of these, 201 had preoperative CT-staging performed. Any patients with visible metastases were excluded, as were those patients who had undergone any preoperative radiotherapy or chemo-radiotherapy. For each eligible patient, information on the patient's age, sex and tumour site was recorded. Carcinomas were staged pathologically according to the Royal College of Pathologists guidelines (Quirke and Williams, 2000) and used the TNM classification (Sobin and Wittekind, 2002) (Table 1). This information was obtained from the original pathology report located in the patient's medical notes. The X-ray filing libraries were searched, and the original preoperative CT scans were retrieved.

\section{Review of CT images}

All available preoperative CT films were reviewed independently by two consultant radiologists (NB and GB), both of whom had been involved in previous CT/MRI comparative studies and were experienced with CT-based staging. Each observer was blinded to the final histological stage and clinical outcome. Images of the primary tumour were evaluated using a dedicated proforma (Figure 1) taking into account patterns of local spread derived from previous histological studies as well as conventional TNM assessment (Sobin and Wittekind, 2002).

$T$ - and $N$-stage The primary tumour was assessed for T-stage. Patients were stratified into 'good' or 'poor' prognosis groups based on CT-predicted T-stage. Early T3 tumours with predicted extramural invasion up to $5 \mathrm{~mm}$ were labelled 'T3good' (corresponding to $\mathrm{pT} 3 \mathrm{a} / \mathrm{pT} 3 \mathrm{~b}$ ). These together with $\mathrm{T} 1 / \mathrm{T} 2$ tumours were stratified into the same favourable prognostic group. More advanced T3 tumours (labelled 'T3bad') that appeared to show extramural invasion more than $5 \mathrm{~mm}$ beyond the muscle coat (corresponding to $\mathrm{pT} 3 \mathrm{c} / \mathrm{pT} 3 \mathrm{~d}$ ) and $\mathrm{T} 4$ tumours with suspected involvement or perforation of the visceral peritoneum or direct invasion of an adjacent organ were considered to have a poor prognosis.

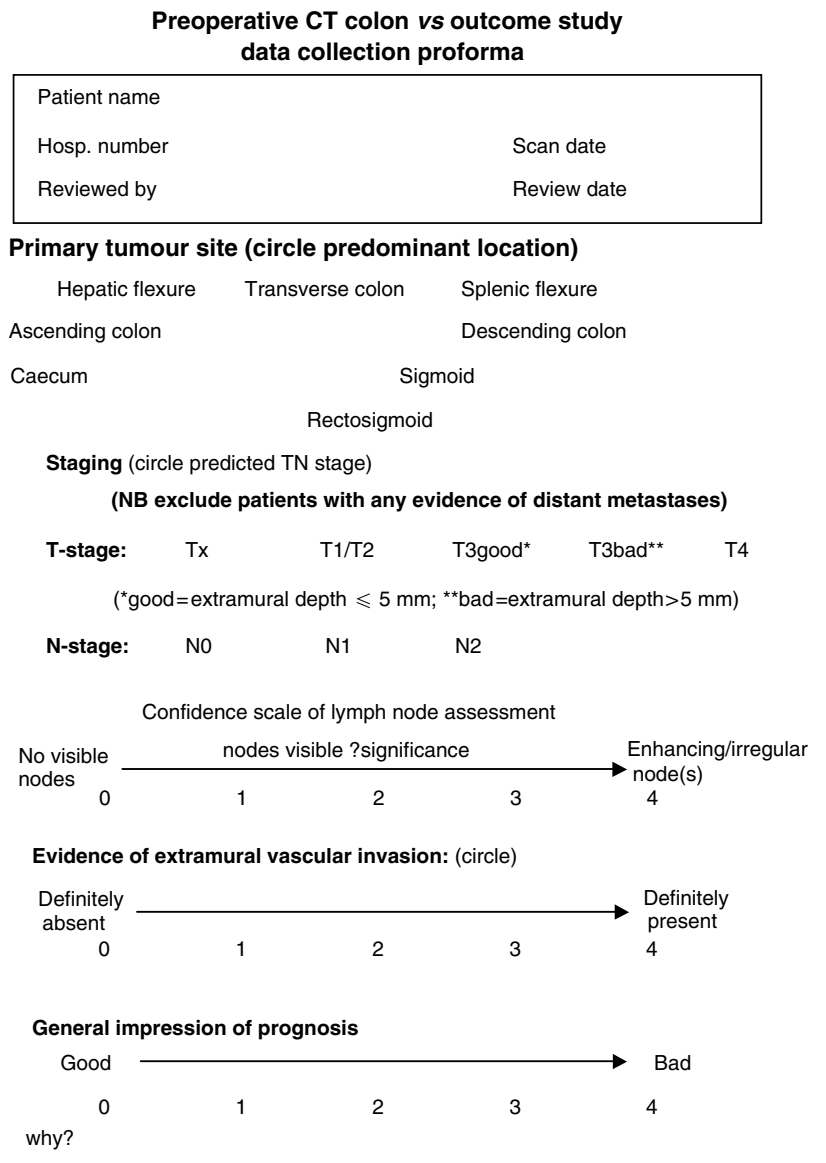

Figure I Data collection proforma for CT colon study.

In addition, the images were assessed for evidence of lymph node involvement. Lymph nodes were considered to be involved by tumour when they were enlarged or had irregular borders. 
Overall 'prognostic score' A five-point linear scale based on assessment of all the radiological features was used in assigning an overall prognostic score (PS) for each patient.

\section{Outcome analysis}

Prospectively compiled clinical follow-up information was recorded from the medical notes. The date and nature of any disease recurrence (local or distant) was documented and if the patient had died, the date and cause of death. Because the median duration of clinical follow-up was relatively short, relapse-free survival (RFS) was chosen as the outcome measure. Death from any cause and recurrence of colorectal cancer or development of a new colorectal primary tumour were counted as events.

Outcome analysis was performed based on each of the following: histological criteria (Table 2); CT-predicted T-stage; CT-defined PS. For each outcome analysis, patients were allocated into one of two prognostic groups ('good' or 'poor').

\section{Statistical analysis}

Kaplan-Meier survival curves were used for interpretation of the outcome data. $\kappa$ coefficients were used in the measurement of interobserver agreement.

\section{RESULTS}

\section{Patient demographics}

During the 5 years between January 2000 and December 2004, 312 patients underwent resection for carcinoma of the colon. Of these, 201 had a preoperative CT scan within a median of 25 days of their operation. Twenty-eight patients had visible metastases on the original CT report, 12 patients had undergone preoperative radiotherapy and one other had an incidental caecal carcinoma resected at laparotomy for an appendix mass. These 40 patients were all excluded. One hundred and twenty six out of $161(78.3 \%)$ scans from 63 men and 63 women were available for review

Table 2 Prognostic categorisation of primary tumour according to histology

\begin{tabular}{|c|c|c|}
\hline Histological variable & 'Good' prognosis & 'Poor' prognosis \\
\hline $\begin{array}{l}\text { T-stage } \\
\mathrm{N} \text {-stage } \\
\text { EMVI } \\
\text { Distant metastases }{ }^{\mathrm{a}} \\
\text { (e.g., peritoneal seedlings) }\end{array}$ & $\begin{array}{c}\text { TI, T2 or T3 } \\
\text { NO } \\
\text { Absent } \\
\text { Absent }\end{array}$ & $\begin{array}{l}\text { T4 } \\
\text { NI or N2 } \\
\text { Present } \\
\text { Present }\end{array}$ \\
\hline
\end{tabular}

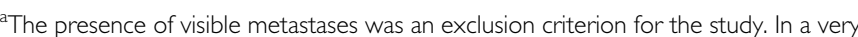
few cases; however, peritoneal seedlings were only identified at operation.
(Figure 2). The median age at operation was 74 years (range 33-89 years).

At the point of survey, 87 patients $(69.0 \%)$ were still alive, with a median follow-up time since operation of 2.68 years (range $=10$ months to 6.25 years).

\section{Accuracy of CT staging}

$\mathrm{T}$ - and $\mathrm{N}$-stage At histopathological examination, seven (5.6\%) of 126 tumours were staged as pT1, $13(10.3 \%)$ as pT2, $67(53.1 \%)$ as pT3 and $39(31.0 \%)$ as pT4.

The overall accuracy of stage-for-stage prediction of T-stage was 60.3 and $60.8 \%$ for observer A and B, respectively (Table 3 ). For the correct recognition of extramural tumour invasion (stage pT3 or pT4), observer A was $83.3 \%$ accurate compared with histology $(92.4 \%$ sensitivity; $42.1 \%$ specificity; positive predictive value (PPV) 89.8\%). Observer B achieved $76.2 \%$ accuracy with

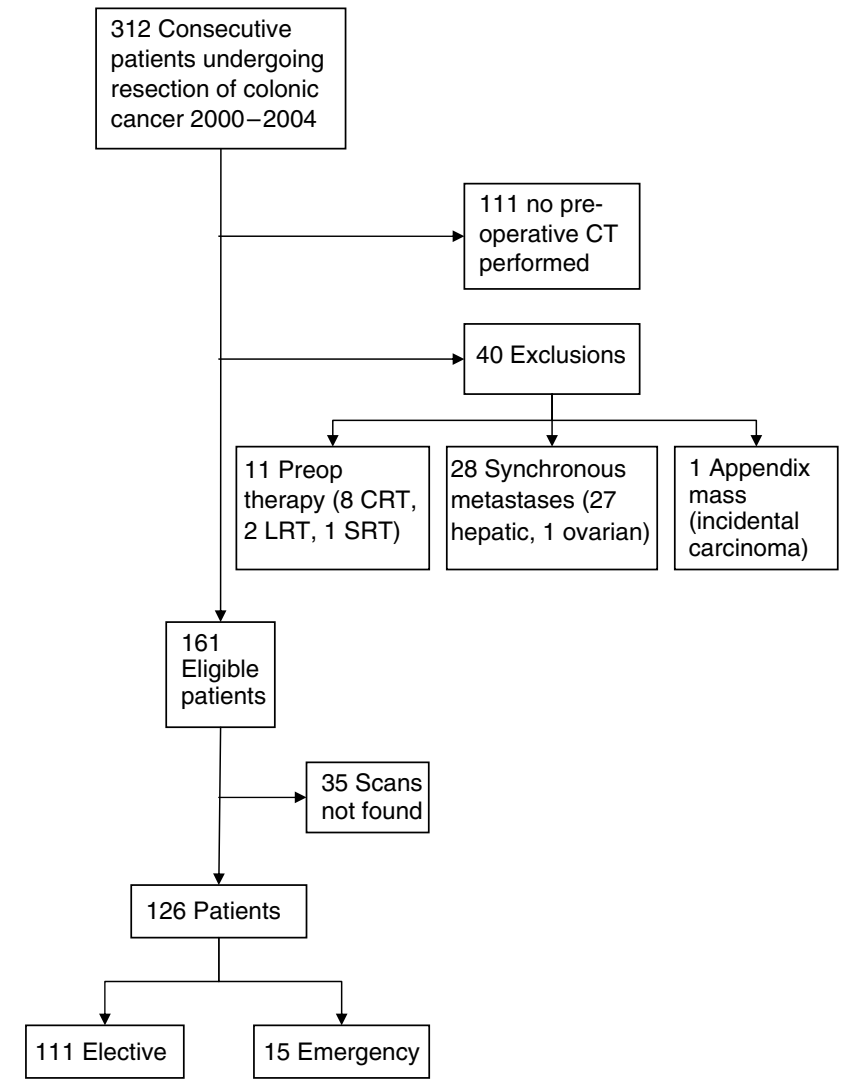

Figure 2 Flowchart for patient selection into study.

Table 3 CT-predicted $\mathrm{T}$ stage for each observer vs histology $\mathrm{T}$ stage

\begin{tabular}{|c|c|c|c|c|c|c|c|c|c|c|c|c|}
\hline Histology & \multicolumn{6}{|c|}{ Observer A CT-predicted T-stage } & \multicolumn{6}{|c|}{ Observer B CT-predicted T stage } \\
\hline T2 & 4 & 6 & 2 & 1 & & 13 & 6 & 1 & 6 & & & 13 \\
\hline T3 & 7 & 26 & 18 & 15 & 1 & 67 & 12 & 14 & 26 & 9 & 6 & 67 \\
\hline T4 & 1 & 4 & 10 & 24 & & 39 & 2 & 4 & 7 & 25 & & 38 \\
\hline Total & 16 & 38 & 30 & 40 & 2 & 126 & 25 & 19 & 39 & 34 & 8 & 125 \\
\hline
\end{tabular}

Observer A: Stage-for-stage accuracy $=60.3 \%$. Extramural invasion (stage T3/T4): Overall accuracy $=83.3 \%$ (sensitivity $=92.4 \%$; specificity $=42.1 \%$ ). Positive predictive value $=89.8 \%$; negative predictive value $=50.0 \%$. Observer B: Stage-for-stage accuracy $=60.8 \%$. Extramural invasion (stage $\mathrm{t} 3 / \mathrm{t} 4)$ : Overall accuracy $=76.2 \%($ sensitivity $=85.9 \%$; specificity $=61.1 \%$. Positive predictive value $=92.4 \%$; negative predictive value $=44.0 \%$. 
sensitivity, specificity and PPV of $85.9,61.1$ and 92.4\%, respectively. Figures 3-6 show examples of CT scans evaluated.

Eighty-six $(68.3 \%)$ of 126 tumours were staged by pathology as pN0, $27(21.4 \%)$ as $\mathrm{pN} 1$ and $13(10.3 \%)$ as $\mathrm{pN} 2$. The overall accuracy of stage-for-stage prediction of $\mathrm{N}$ stage was poor $(50.4$ and $54.8 \%$ for observer A and B, respectively), but for lymph node status (involved or tumour-free), accuracy was slightly better at 61.8 and $62.1 \%$ for each observer.

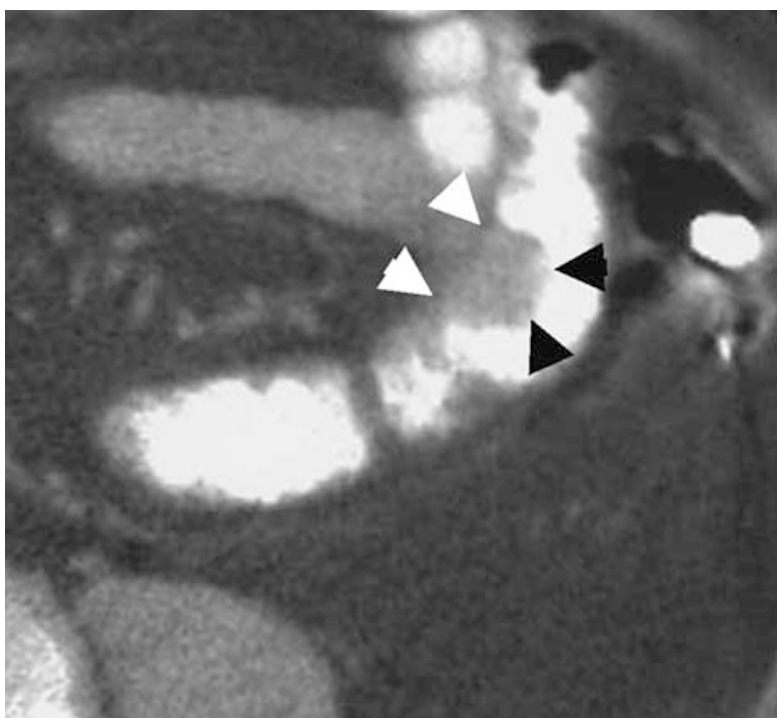

Figure 3 Preoperative CT scan showing a polypoid tumour of the descending colon extending into the lumen (black arrowheads). Tumour does not extend beyond the contour of the muscle coat indicating that this is an early $\mathrm{TI} / \mathrm{T} 2$ tumour. Pathological staging was $\mathrm{PT} 2$.

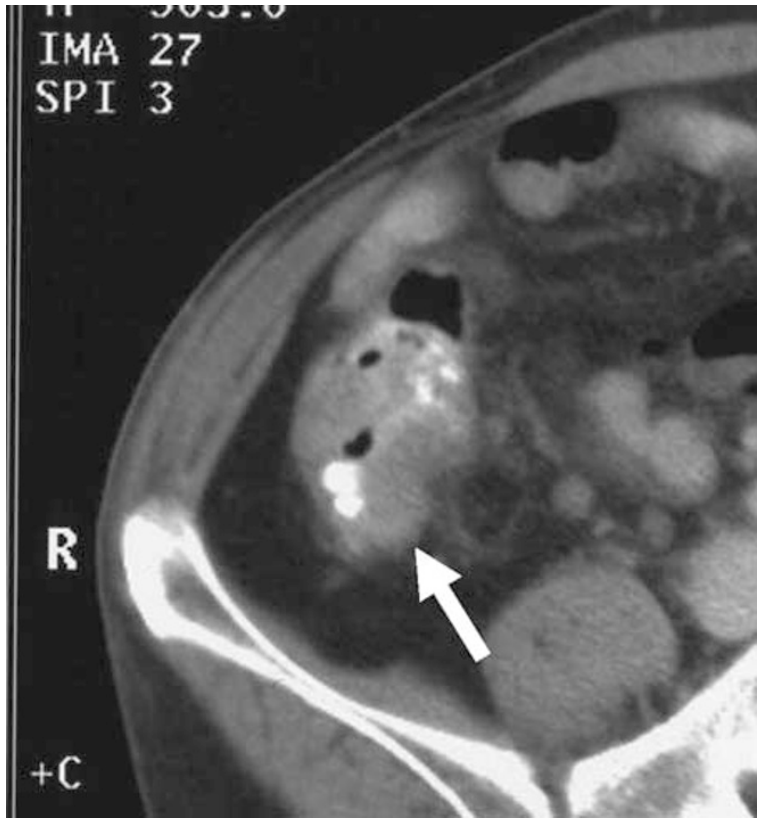

Figure 4 Preoperative CT scan showing a fungating tumour of the ascending colon. The colonic wall is thickened and the posterior contour is irregular owing to tumour projection beyond the non-peritonealised muscle coat. As there is no tumour involvement of the peritonealised surfaces, this is considered a relatively good prognosis T3 tumour. Pathology confirmed a PT3 tumour of the ascending colon.
Using the criteria described in Table 2, patients were categorised as having either 'good' or 'poor' prognosis tumours based on their histological staging. Seventy-one out of $126(56.3 \%)$ patients had tumours categorised histologically as poor prognosis.

Prognostic group based on CT T-stage vs histology The best correlation between CT-predicted T-stage prognostic group and histology-predicted group showed an overall accuracy of $71 \%$ (sensitivity 73\%; specificity 67\%), with a PPV (for 'poor' prognosis) of $74 \%$. There was $79.2 \%$ agreement $(\kappa=0.59)$ between the observers on reporting of advanced T3c/d and T4 tumours (Table 4).

Prognostic group based on overall 'PS' vs histology The subjective PS $(0-4)$ was also used to assign patients into prognostic categories, with $\mathrm{PS}=0-2$ corresponding to a 'good' PS, and $\mathrm{PS}=3-4$ a 'bad' score. Correlating PS-based prognostic group with histology-based group, observer A achieved the highest

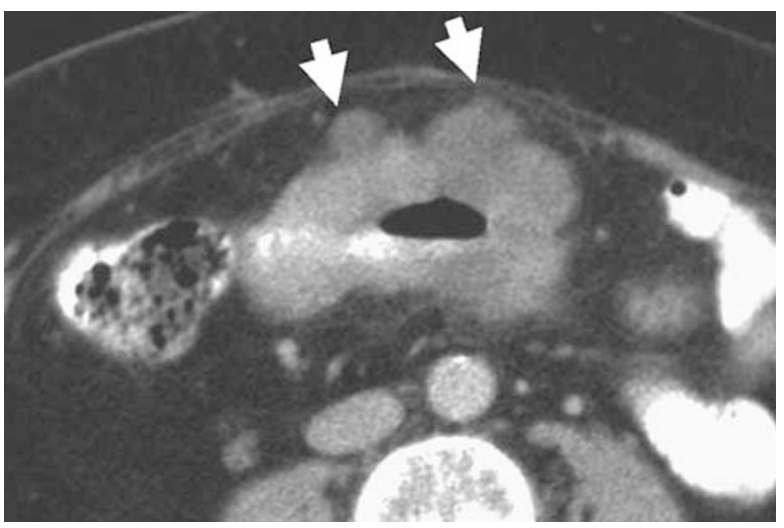

Figure 5 Preoperative CT scan showing a fungating tumour of the transverse colon. The anterior colonic wall is distorted by tumour. As there is minimal pericolic fat and the colon is peritonealised at this location, there is a very high probability that the tumour will be stage T4. Pathological staging was $\mathrm{PT} 4 \mathrm{pNI}$

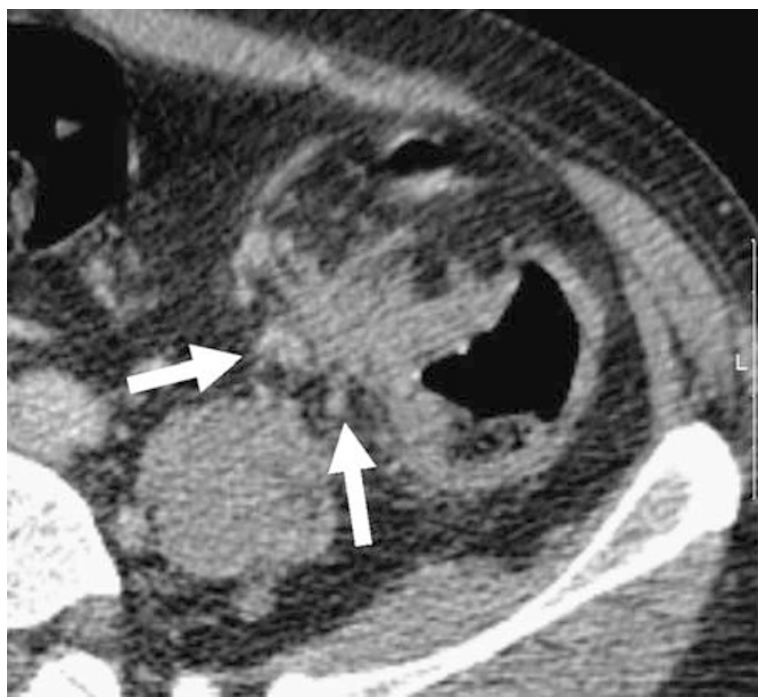

Figure 6 Preoperative CT scan showing a bulky tumour of the descending/sigmoid junction. There is irregular nodular extension medially (arrows) indicating T3 infiltration. This is likely to extend through the medial nonperitonealised, mesenteric surface of the colon. The pathological stage was confirmed as PT3. 
Table 4 Agreement of CT-predicted good/poor prognosis $T$ stage (Observer A vs observer B)

Observer A

\begin{tabular}{lcc}
\cline { 2 - 3 } Observer B & TI/2, T3good & T3bad, T4 \\
\hline TI/2, T3good & 48 & 21 \\
T3bad, T4 & 5 & 51 \\
\hline
\end{tabular}

Inter-observer agreement $=79 \%(\kappa=0.59)$.

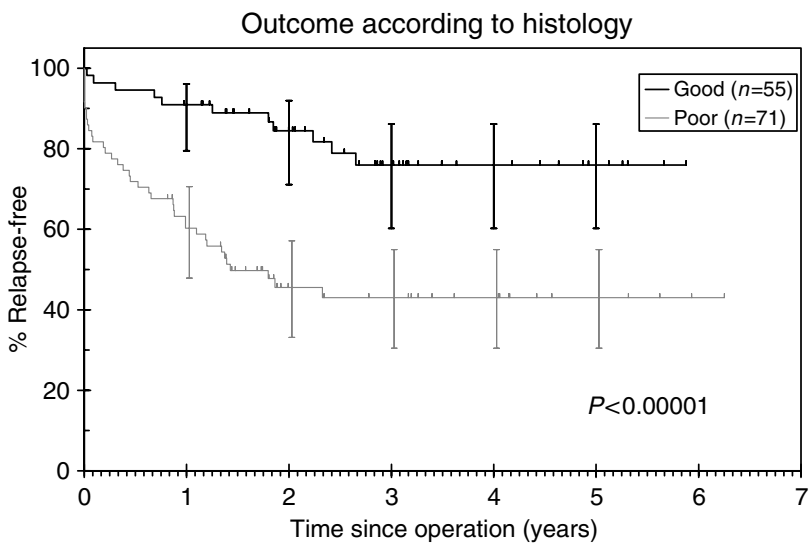

Figure 7 Kaplan-Meier RFS curves according to histological group.

'accuracy' of $69 \%$ (sensitivity $73 \%$; specificity $64 \%$ ), with a PPV (for 'poor' prognosis) of $72 \%$. Inter-observer agreement for PSbased prognostic group was $80 \%(\kappa=0.60)$.

\section{Prediction of outcome}

Kaplan-Meier survival curves were constructed to illustrate differences in outcome between prognostic groups. As the median length of recorded follow-up was just under 3 years, RFS was chosen as the preferred outcome measure.

Histology Three-year RFS for patients with 'good' prognosis tumours according to histology was 75\% (95\% confidence interval (CI): $60-86 \%)$ vs $43 \%(30-45 \%)$ for 'poor' prognosis tumours based on histology $(P<0.00001$, Figure 7$)$.

Predicted CT T-stage Three-year RFS for patients with CT-based 'good' prognosis tumours was 71\% (95\% CI: 55-82\%) compared with $43 \%(34-60 \%)$ in 'poor' tumours for observer A $(P=0.0066)$. This compared with $66 \%(51-75 \%)$ vs $49 \%$ (34-63\%) for observer B $(P=0.0475$, Figure 8$)$.

Overall CT PS Three-year RFS for patients with 'good' PS (0-2) was $70 \%$ (95\% CI: $54-81 \%$ ) compared with $48 \%(35-60 \%)$ for patients with 'poor' PS (3-4) for observer A $(P=0.0091$, Figure 9). This compared with $63 \%(50-74 \%)$ vs $52 \%$ (37-65\%) for observer B $(P=0.1655)$.

\section{DISCUSSION}

Knowledge of the extent of disease at initial diagnosis is critical for the proper management of patients with colorectal cancer. Several authors have found that preoperative CT provides useful information in up to half and definitely alters clinical management
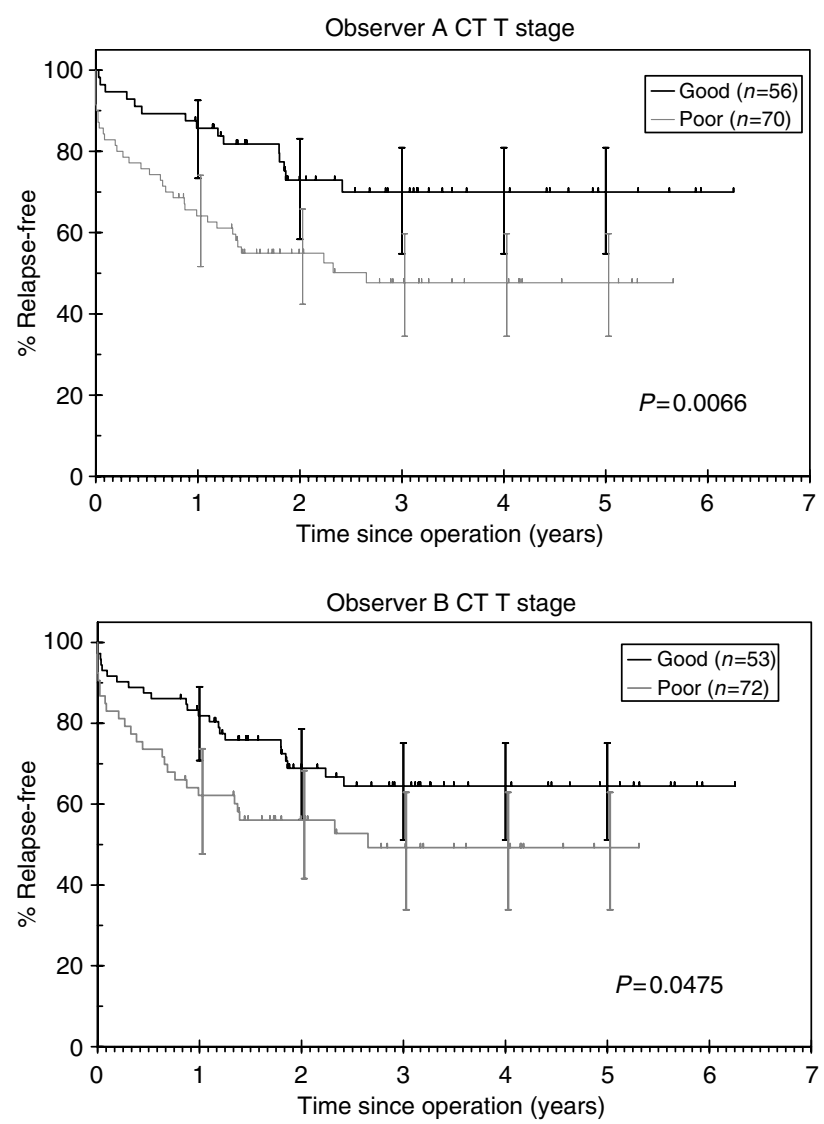

Figure 8 Kaplan-Meier RFS curves according to CT-predicted T-stage.

in around $20 \%$ of patients with colon cancer (Kerner et al, 1993 Barton et al, 2002; Mauchley et al, 2005) and most often this relates to the detection of liver metastases. In our series, we excluded patients with preoperatively detected distant metastases in order to investigate whether, contrary to conventional wisdom, preoperative CT staging could be useful in predicting outcome in the majority of patients who do not have metastatic disease. No other published series has compared CT-predicted tumour stage with clinical outcome.

We were careful to apply the accepted pathological TNM definitions of T-stage (Table 1) to CT staging. The overall accuracy of T-stage prediction by each of our observers was 60.3 and $60.8 \%$ compared with histology, whereas identification of T3/T4 tumours was $85.9-92.4 \%$ sensitive, with a PPV of at least $90-92 \%$. These results appear to compare favourably with other studies, which have reported sensitivities of between 55 and $61 \%$ and specificities of $67-81 \%$ in the detection of 'serosal', 'extramural' or 'local (T3T4) invasion (Freeny et al, 1986; Acunas et al, 1990; Zerhouni et al, 1996). However, this relative lack of definition of depth of invasion makes direct comparison between those studies and our own results difficult. Newer multi-slice/spiral-CT scanners, which are able to collect $1-\mathrm{mm}$ thick slices, within a single breath hold permitting 3D reconstruction, give improved resolution and image quality. Two small series using CT colonography ('virtual colonoscopy') have produced good results, with correct T-staging reported in $26(78.7 \%)$ of 33 and $30(81 \%)$ of 37 colorectal tumours (Hundt et al, 1999; Laghi et al, 2002).

The reported sensitivities for lymph node detection range between 19 and 97\% (Freeny et al, 1986; Acunas et al, 1990; Zerhouni et al, 1996; McAndrew and Saba, 1999; Laghi et al, 2002). 

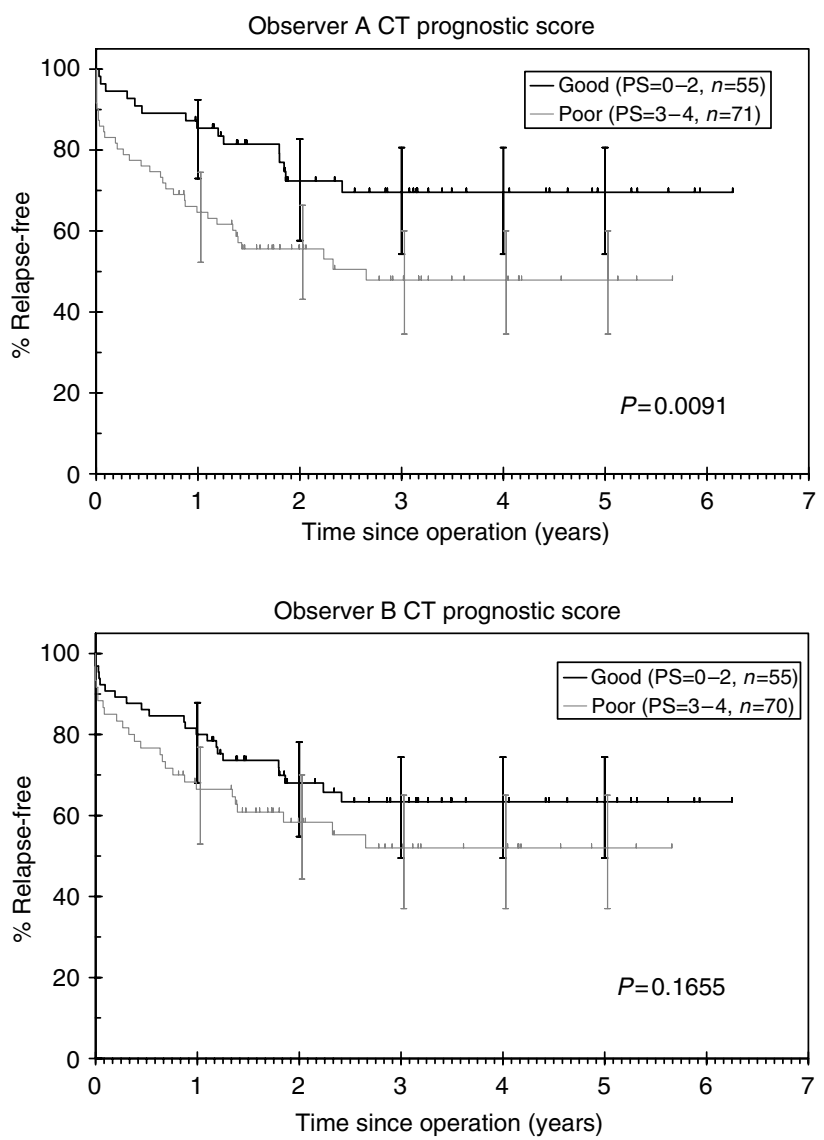

Figure 9 Kaplan-Meier RFS curves according to CT-predicted overall PS

The difficulties of staging lymph nodes based on size criteria are well known - small/normal-sized nodes may have microscopic tumour infiltration, whereas, occasionally, very large nodes may be reactive, rather than malignant. In our series, lymph node status was accurately predicted by each of the two observers in 61.8 and $62.1 \%$, respectively. Consequently, we have not found CT to be particularly useful in the assessment of lymph nodes.

Most published series have emphasised the value of preoperative CT staging to diagnose metastatic disease (Freeny et al, 1986; Balthazar et al, 1988; Acunas et al, 1990; Kerner et al, 1993; Thoeni, 1997). Many have concluded that preoperative CT staging is of little value other than to exclude distant metastases because of its limited accuracy, particularly in staging early tumours (Thoeni, 1997). Clearly, no imaging modality that evaluates gross morphology (such as CT) will ever be as 'accurate' as the microscope. Nevertheless, although it is undoubtedly true that CT does not 'accurately' measure depth of invasion in early-stage tumours, this is not usually of great clinical significance. The clinical outcome for patients with early-stage (T1-2, N0) colonic tumours is usually excellent (5-year overall survival 80-95\%). In contrast, patients with locally advanced T4 tumours have a much poorer outcome and some histological studies have also identified extramural invasion greater than $5 \mathrm{~mm}(\mathrm{~T} 3 \mathrm{c} / \mathrm{d})$ as a poor prognostic feature (Merkel et al, 2001) and we have recognised this in our categorisation of CT-predicted T-stage. Currently, patients with poor prognostic features on histology may be offered postoperative adjuvant systemic chemotherapy. Identification of patients with locally advanced colonic tumours preoperatively would be of great importance if preoperative neo-adjuvant therapies were to be considered. We have applied the lessons learnt from using MRI to assess the surgical anatomy of the rectum to our CT-based assessment of colonic tumours. Knowledge of the surgical anatomy of the colon and specifically the distribution of peritonealised and nonperitonealised surfaces has enabled us to improve our assessment of the depth of invasion, the presence of $\mathrm{T} 4$ disease (peritoneal perforation) and potential resectability.

This study is interesting because it highlights the heterogeneity of actual clinical outcomes for different pathological stages. Pathological criteria are widely accepted as the basis for prognostication (and therefore as an indication for adjuvant therapy) because there is an abundance of evidence that correlates pathology with outcome for populations. However, within a population of patients, some with 'good' prognosis tumours will relapse and some with 'poor' prognosis tumours will do well, having been cured by surgery alone, receiving no extra benefit from their adjuvant chemotherapy. In our study, the RFS at 3 years for patients with no adverse pathological features was 75 compared with $43 \%$ for those with one or more poor prognosis feature $(P<0.00001)$. There is to date no other published study that has directly compared preoperative CT staging of the primary tumour with clinical outcome, but our study does appear to show that CT can also discriminate between 'good' and 'poor' prognosis tumours, with similar 'accuracy' to pathology. Not every 'poor' tumour relapses and not every 'good' tumour remains disease free, but an overall trend is seen in the population and the 3-year RFS based on CT predicted T-stage criteria was 71 vs $43 \%(P<0.0066)$. The fact that the CT-stage may not correlate exactly to the pathological stage is therefore of less importance as the CT stage predicts outcome as well as pathology for the population.

We attempted to produce an overall PS taking into account all visible features (namely $\mathrm{T}$-stage, nodal status and extramural vascular invasion). We found that this was actually less discriminating than CT-predicted T-stage alone, and the prognostic accuracy of T-stage alone was diluted, rather than enhanced by the combination of other features. We believe that the inaccuracy of CT assessment of lymph node status was the major contributory factor, together with the lack of precise objectivity in defining the criteria for the five-point PS, which would limit reproducibility. We therefore do not recommend the use of such a score in its present form.

High sensitivity rates are desirable to enable the potential therapeutic benefit of preoperative treatments to be offered to as many appropriate individuals as possible. However, particularly from the point of view of clinical trials, high PPVs are of even greater importance to avoid giving unnecessary, potentially toxic treatments to patients, who would not otherwise be offered them, even in the usual post-operative setting. Our study has shown high sensitivity (86-92\%) and PPVs (90-92\%) for the identification of T3 and T4 tumours by preoperative CT. Furthermore, there is reasonable correlation between CT-predicted 'poor prognosis' $\mathrm{T}$-stage (i.e. $\mathrm{T} 4$ and $\mathrm{T} 3 \mathrm{c}$ and $\mathrm{d}$ ) and poor histological group $(\mathrm{PPV}=74-75 \%)$. However, the most compelling evidence for CTbased prognostication comes from the survival analysis. If CTpredicted T-stage discriminates between good and poor outcome as well as the accepted pathological criteria (which it does in this study), then it seems no more unreasonable to use preoperative CT to guide preoperative treatment, as it does to use pathological criteria to indicate post-operative therapy. The benefits of preoperative therapy in colon cancer, although theoretically persuasive (potential downstaging, greater tolerability), remain unproven. Reliable preoperative staging is required before any pre$v s$ post-operative chemotherapy trial could be set up, and the results of this relatively small study would require further validation in a multi-centre setting. Nonetheless, our results suggest that preoperative CT may be of greater use than simply the exclusion of distant metastases.

In conclusion, we have demonstrated that preoperative CT can predict the clinical outcome for 'good' and 'poor' prognosis 
tumours with the same accuracy as histopathology. Our results suggest that it is therefore a robust method for stratifying patients preoperatively, and recognition of poor prognosis tumours preoperatively may permit investigation into the use of neoadjuvant therapy in colon cancer. This will form the basis of a future clinical trial.

\section{REFERENCES}

Acunas B, Rozanes I, Acunas G, Celik L, Sayi I, Gokmen E (1990) Preoperative CT staging of colon carcinoma (excluding the rectosigmoid region). Eur J Radiol 11: 150 - 153

Balthazar EJ, Megibow AJ, Hulnick D, Naidich DP (1988) Carcinoma of the colon: detection and preoperative staging by CT. AJR Am J Roentgenol 150: $301-306$

Barton JB, Langdale LA, Cummins JS, Stelzner M, Lynge DC, Mock CN, Nason KS, Billingsley KG (2002) The utility of routine preoperative computed tomography scanning in the management of veterans with colon cancer. Am J Surg 183: 499-503

Dukes CE (1932) The classification of cancer of the rectum. J Path Bact 35 $323-332$

Freeny PC, Marks WM, Ryan JA, Bolen JW (1986) Colorectal carcinoma evaluation with CT: preoperative staging and detection of postoperative recurrence. Radiology 158: 347-353

Hundt W, Braunschweig R, Reiser M (1999) Evaluation of spiral CT in staging of colon and rectum carcinoma. Eur Radiol 9: 78-84

Kerner BA, Oliver GC, Eisenstat TE, Rubin RJ, Salvati EP (1993) Is preoperative computerized tomography useful in assessing patients with colorectal carcinoma? Dis Colon Rectum 36: 1050- 1053

Laghi A, Iannaccone R, Trenna S, Mangiapane F, Sinibaldi G, Piacentini F, Sammartino P, Stipa V, Passariello R (2002) Multislice spiral CT colonography in the evaluation of colorectal neoplasms. Radiol Med (Torino) 104: $394-403$

Mauchley DC, Lynge DC, Langdale LA, Stelzner MG, Mock CN, Billingsley KG (2005) Clinical utility and cost-effectiveness of routine preoperative

\section{ACKNOWLEDGEMENTS}

We thank Sarah Burton for establishing the original Mayday Hospital colorectal cancer database, and Barbara Mason (Royal Marsden Hospital) and Shanti Deefholts (Mayday University Hospital) for their assistance in retrieving CT films from filing.

computed tomography scanning in patients with colon cancer. Am J Surg 189: 512 - 517; discussion 517.

McAndrew MR, Saba AK (1999) Efficacy of routine preoperative computed tomography scans in colon cancer. Am Surg 65: 205-208

Merkel S, Mansmann U, Siassi M, Papadopoulos T, Hohenberger W, Hermanek P (2001) The prognostic inhomogeneity in pT3 rectal carcinomas. Int J Colorectal Dis 16: 298-304

Northern Ireland Cancer Registry (2005) Cancer Incidence and Mortality Office for National Statistics (2005) Cancer Statistics Registrations: Registrations of Cancer diagnosed in 2002, England. In Series MB1 No. 33. London: National Statistics

Quirke P, Williams GT (2000) Minimum dataset for colorectal cancer histopathology reports. London: The Royal College of Pathologists

Scottish Cancer Registry (2005) Cancer in Scotland. Scotland: NHS National Services

Sobin LH, Wittekind C (2002) TNM Classification of Malignant Tumours. (UICC) 6th Edition. Hoboken, New Jersey: JohnWiley \& Sons

Thoeni RF (1997) Colorectal cancer. Radiologic staging. Radiol Clin North Am 35: 457-485

Welsh Cancer Intelligence and Surveillance Unit (2006) Cancer Incidence in Wales 1992-2003

Zerhouni EA, Rutter C, Hamilton SR, Balfe DM, Megibow AJ, Francis IR, Moss AA, Heiken JP, Tempany CM, Aisen AM, Weinreb JC, Gatsonis C, McNeil BJ (1996) CT and MR imaging in the staging of colorectal carcinoma: report of the Radiology Diagnostic Oncology Group II. Radiology 200: $443-451$ 